\title{
An unusual association of mirizzi syndrome and cholangiocarcinoma - a case report
}

\section{Summary}

A 51-year-old male presented with painless obstructive jaundice with loss of appetite and weight. MRI abdomen showed Mirizzi syndrome and after eventually undergoing cholecystectomy the histological diagnosis of cholangiocarcinoma was made on the pathological specimen. The concurrent presence of these two relatively rare diseases can be explained on the basis of the similar pathogenesis - Chronic inflammation.
Volume 4 Issue 4 - 2016

\begin{abstract}
Lubna Bashir Munshi, Sayee Sundar Alagusundaramoorthy, Isha Verma

Department of Internal Medicine, Monmouth Medical Center, Long Branch, USA
\end{abstract}

\author{
Correspondence: Lubna Bashir Munshi, Department of \\ Internal Medicine, Monmouth Medical Center, Second Ave, Long \\ Branch, NJ, USA, Tel (732) 9235000 , \\ Email lubnabashirmunshi@gmail.com
}

Received: May 23, 2016 | Published: June 23, 2016

\section{Introduction}

Mirizzi syndrome is a rare complication of symptomatic gall stone disease that occurs when the common hepatic duct is extrinsically obstructed by an impacted stone in the cystic duct at the neck of the gall bladder ${ }^{1}$ Pablo Luis Mirizzi ${ }^{2}$ first described it in 1948 \& four types of Mirizzi syndrome have been recognized until now to aid in the appropriate treatment. ${ }^{3}$ Symptoms and signs are similar to gall stone causing obstruction with the clinical triad of Fever, Right upper quadrant pain and Obstructive pattern jaundice. The obstruction of the $\mathrm{CBD}$ and the obstructive jaundice pattern seen in Mirizzi syndrome is thought to be related to the external compression and fibrotic changes due to the presence of chronic inflammation. ${ }^{4}$ A number of reports have been published in the literature showing a clear association between GB carcinoma and Mirizzi syndrome ${ }^{5,6}$ however our case describes the presence of Cholangiocarcinoma with this unusual syndrome. The main factor thought to play a key role in the pathogenesis of both these diseases is the presence of chronic biliary inflammation. Our case describes a patient with Mirizzi syndrome, who showed postoperative pathological findings consistent with Cholangiocarcinoma.

\section{Case presentation}

A 51-year-old Caucasian male presented to our emergency department with yellow skin, eyes and light colored stools which had developed over 2-3days along with some loss of appetite \& weight. He was not complaining of any pain or change in his bowel or bladder habits. There was no associated fever, pain in the abdomen, nausea/vomiting or pruritus. His past medical history was significant for Diabetes Mellitus and Hyperlipidemia, which were being managed medically. The patient only admitted to occasional social drinking. Labs revealed an Obstructive Jaundice pattern with direct hyperbilirubinemia and elevated liver enzymes. Previous records showed normal LFTs despite statin therapy for Hyperlipidemia. US abdomen showed presence of gallstones and GB sludge. CT Abdomen done in the emergency room showed multiple calcified gallstones within the Gall Bladder and a large gallstone at the neck of the Gall Bladder (Figure 1). There was also mild intrahepatic biliary ductal dilatation noted in the CT scan. The hepatitis panel was non-reactive for Hep A, B and C, ANA was negative and SMA was 1:20. Further labs revealed an elevated CA 19-9 level. Working diagnosis at this time involved choledocholithiasis with autoimmune etiology vs Mirizzi syndrome MRCP showed a large GB stone within the neck of the gall bladder with asymmetric biliary ductal dilatation. MRI abdomen (Figure 2) confirmed the GB neck gallstone and showed the stone exerting the mass effect on the adjacent $\mathrm{CBD}$ contributing to the dilatation of the intrahepatic biliary system superior to the level of the obstruction consistent with Mirizzi syndrome type I.

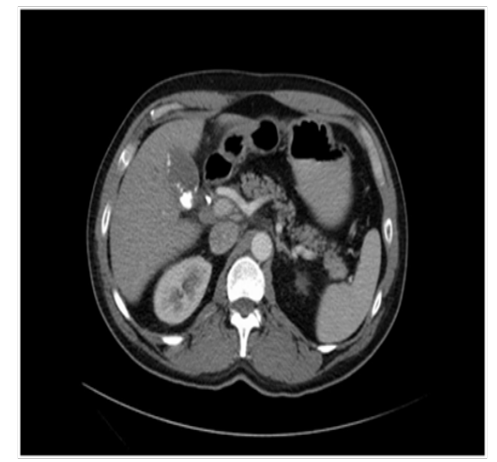

Figure I CT scan image showing the obstructing gall stone at the neck of the gall bladder.

Emergent ERCP (Figure 3) was done and findings were consistent with the Mirizzi syndrome type I and also showed a biliary stricture in the distal right intrahepatic system extending to the common hepatic duct. The right intrahepatic system was stented at the level of the right intrahepatic and there was dilatation noted on the left side. The patient underwent a follow up cholecystectomy during which intraoperative findings showed an edematous \& non-gangrenous GB. The cholecystectomy specimens and the biopsy of the CBD strictures were sent to pathology for histo-pathological correlation.

The patient underwent a repeat ERCP after the surgery for stent removal, replacement and evaluation of the left sided dilation along with cytology brushing and biliary biopsy. It was also noted that the gallstone had extended up into the porta hepatis and there was extensive adherence of the cystic duct with the stone to the common hepatic 
duct at that location along with a hilar stricture. After microscopic examination the histo-pathological diagnosis of adenocarcinoma with the mucinous component of the CBD stricture was made.

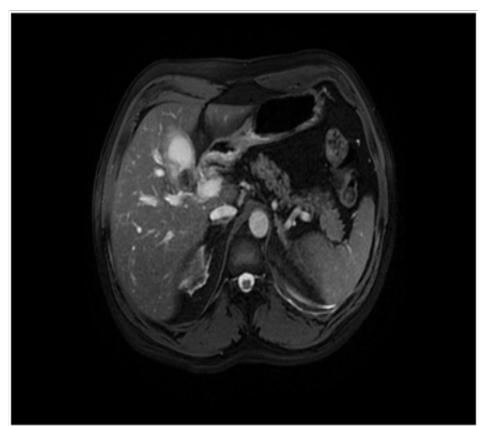

Figure $2 \mathrm{MRI}$ image showing the obstructing gall stone at the neck of the gall bladder.

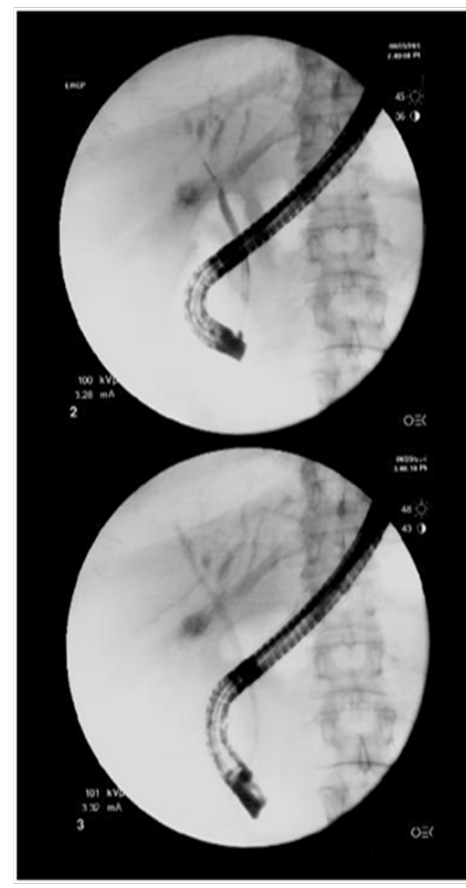

Figure 3 ERCP image.

The final diagnosis of Cholangiocarcinoma was made and was seen in association with Mirrizi syndrome. Patient was started on chemotherapy due to the presence of Cholangiocarcinoma. Further follow up revealed that the treatment regimen was unsuccessful and the patient developed liver as well as splenic metastasis. The patient also became hypercoagulable and had bilateral PE, right hepatic artery occlusion and splenic infarcts. Patient was re-admitted because of the above and was placed an IVC filter and Anti-coagulation. However overall, his condition and prognosis remained poor and he finally expired from all the complications of the metastatic Cholangiocarcinoma

\section{Discussion}

Cholangiocarcinoma, the most common biliary tract malignancy, is a relatively rare neoplasm with a reported incidence rate of 1.2 cases per 100,000 in the western world and a 5year survival of less than $5 \%$ in persons suffering from advanced stages of the disease. Throughout the last decade the incidence of CCA has been steadily increasing. ${ }^{7}$ The tumor arises from the epithelial cells of the biliary tree and in addition to well-established risk factors; novel possible risk factors have been identified that are of high importance due to the increasing incidence. CCA tends to develop in the background of chronic inflammation and cholestasis. ${ }^{8,9}$ Different factors that have been identified to promote chronic inflammation include liver fluke infestation, viral hepatitis and exogenous nitrosamine mediated DNA damage, which have also been linked to CCA genesis. ${ }^{10}$ The risk factors and underlying complex mechanisms leading to the development of CCA is poorly understood and it's therefore essential that these risk factors be identified at the earliest to devise a targeted therapy and effective treatment. ${ }^{11} \mathrm{CCA}$ is known to have a very poor prognosis due to delayed diagnosis and therefore the loss of effective treatment strategies to overcome the tumor burden.

Mirizzi syndrome is a rare complication that occurs with symptomatic gall stone disease explained as an inflammatory phenomenon secondary to a pressure ulcer caused by an impacted gallstone at the gall bladder Infundibulum. ${ }^{12,13}$ Reported incidence varies from $1 \%$ in developed countries to 4.7 to $5.7 \%$ in developing countries. ${ }^{1}$ The impacted gallstone together with inflammatory response causes external obstruction of the bile duct, eroding into the duct evolving into a cholecystocholedochal or cholecystohepatic fistula with different degrees of communication between gall bladder and bile duct. ${ }^{14}$ Mirizzi syndrome has been shown to be associated with GB carcinoma with a reported incidence of $6 \%$ in $28 \%$ cases diagnosed with Mirizzi syndrome. The treatment of Mirizzi syndrome depends on the type at presentation and it varies from simple cholecystectomy to roux-e-y ansostomsis for a bilio-enteric fistula. ${ }^{15}$

Although a clear relationship has been established that presence of hepatolithiasis as a contributing factor to the development of Mirizzi syndrome, recent reports have suggested that cholangitis, cholecystitis and cholelithiasis are also associated with an increased risk of cholangiocarcinoma. ${ }^{16}$ The pathogenesis is though to be mediated by the chronic inflammation that is associated with all these conditions. Our report describes the presence of Mirizzi syndrome in a patient and the diagnosis of CCA in the post-surgical specimen. The concurrent presence of these two unusual diseases is thought to be secondary to the common etiological factor - Chronic inflammation resulting in the occurrence of both these diseases in the same patient. Though a clear association has been established regarding the presence of GB carcinoma in patients with Mirizzi Syndrome, there are no studies evident in the literature regarding its association with other tumors. Further studies are warranted in this area as the presence of chronic inflammation in the cellular micro-environment can not only lead to the formation of ulcers but also to tumors. ${ }^{17}$

\section{Conclusion}

Mirizzi syndrome, characterized by the inflammatory response causing external obstruction of the bile duct has also been shown to be associated with cholangiocarcinoma in our patient. The approach to patients with suspected Mirizzi syndrome should be individualized on patient basis. A complete preoperative diagnosis should be made with all investigative modalities available, and if encountered during surgery every effort should be made to perform an accurate and cautious surgery trying to identify the type of Mirizzi and perform the most adequate treatment for each particular case. Further studies are also warranted to study the association between this syndrome and other type of tumors. 


\section{Acknowledgements}

None.

\section{Conflict of interest}

The author declares no conflict of interest.

\section{References}

1. Beltrán MA. Mirizzi syndrome: history, current knowledge and proposal of a simplified classification. World J Gastroenterol. 2012;18(34):4639 4650 .

2. Leopardi LN, Maddern GJ. Pablo Luis Mirizzi: the man behind the syndrome. ANZ J Surg. 2007;77(12):1062-1064.

3. Csendes A, Díaz JC, Burdiles P, et al. Mirizzi syndrome and cholecystobiliary fistula:a unifying classification. Br J Surg. 1989;76(11):11391143 .

4. Pemberton M, Wells AD. The Mirizzi syndrome. Postgrad Med J. 1997;73(862):487-490.

5. Prasad TL, Kumar A, Sikora SS, et al. Mirizzi syndrome and gallbladder cancer. J Hepatobiliary Pancreat Surg. 2006;13(4):323-326.

6. Ramia JM, Villar J, Muffak K, et al. Ferron JA. Mirizzi syndrome and gallbladder cancer. Cir Esp. 2007;81(2):105-106.

7. Charbel H, Al-Kawas FH. Cholangiocarcinoma:epidemiology, risk factors, pathogenesis, and diagnosis. Curr Gastroenterol Rep. 2011;13(2):182-187.

8. Leyva-Illades D, McMillin M, Quinn M, et al. Cholangiocarcinoma pathogenesis: Role of the tumor microenvironment. Transl Gastrointest Cancer. 2012;1(1):71-80.

9. Landskron G, DelaFuente M, Thuwajit P, et al. Chronic inflammation and cytokines in the tumor microenvironment. $J$ Immunol Res. 2014;2014:149185.

10. Bragazzi MC, Cardinale V, Carpino G, et al. Cholangiocarcinoma: Epidemiology and risk factors. Transl gatrointest cancer. 2012;1(1):21-32.

11. Yip AW, Chow WC, Chan J, et al. Mirizzi syndrome with Cholecystocholedochal fistula: preoperative diagnosis and management. Surgery. 1992;111(3):335-338.

12. Dorrance HR, Lingam MK, Hair A, et al. Acquired abnormalities of the biliary tract from chronic gallstone disease. J Am Coll Surg. 1999;189(3):269-273.

13. Corlette MB, Bismuth H. Biliobiliary fistula. A trap in the surgery of cholelithiasis. Arch Surg. 1975;110(4):377-383.

14. Krhenbhl L, Moser JJ, Redaelli C, et al. A standardized surgical approach for the treatment of Mirizzi syndrome. Dig Surg. 1997;14:272-276.

15. Tyson GL, El-Serag HB. Risk Factors of Cholangiocarcinoma. Hepatology. 2011;54(1):173-184.

16. Chang JS1, Tsai CR, Chen LT. Medical risk factors associated with cholangiocarcinoma in Taiwan: a population-based case-control study. PLoS One. 2013;22;8(7):e69981.

17. Futoshi Okada*† Inflammation-related carcinogenesis: current findings in epidemiological trends, causes and mechanisms yonago. Acta Med. 2014;57(2):65-72. 